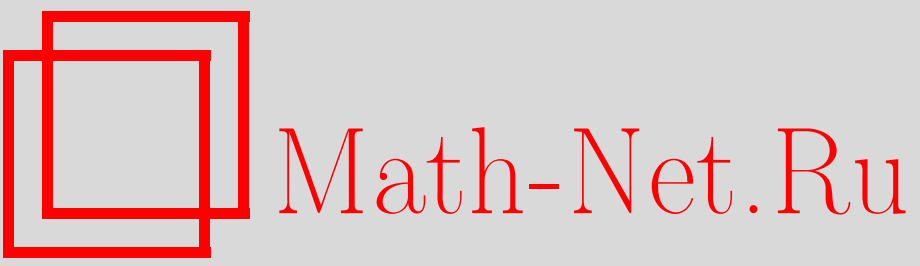

А. С. Запов, Нелинейный панельный флаттер. Задача Болотина при наличии вязкого трения, Итоги науки и техн. Сер. Соврем. мат. и ее прил. Темат. обз., 2020, том 186, 45-51

DOI: https://doi.org/10.36535/0233-6723-2020-186-45-51

Использование Общероссийского математического портала Math-Net.Ru подразумевает, что вы прочитали и согласны с пользовательским соглашением

http://www.mathnet.ru/rus/agreement

Параметры загрузки:

IP: 52.6 .47 .48

26 апреля 2023 г., 12:05:03 


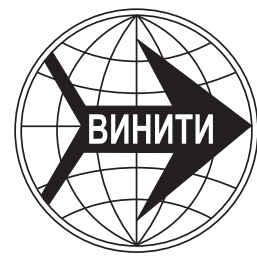

ИТОГИ НАУКИ И ТЕХНИКИ.

Современная математика и ее приложения.

Тематические обзоры.

Том 186 (2020). C. 45-51

DOI: $10.36535 / 0233-6723-2020-186-45-51$

УДК 517.958

\title{
НЕЛИНЕЙНЫЙ ПАНЕЛЬНЫЙ ФЛАТТЕР. ЗАДАЧА БОЛОТИНА ПРИ НАЛИЧИИ ВЯЗКОГО ТРЕНИЯ
}

\author{
(c) 2020 г. $\quad$ A. С. ЗАПОВ
}

\begin{abstract}
АннотАция. Рассматривается нелинейная краевая задача, предложенная в качестве простейшей модели для описания колебания в потоке газа. Изучен вопрос об устойчивости нулевого состояния равновесия, а также найдено критическое значение скорости набегающего потока газа. Найдены точные решения изучаемой задачи в виде периодических по времени функций. Исследован вопрос об устойчивости таких автомодельных периодических решений. Все результаты анализа нелинейной краевой задачи носят аналитический характер. Их вывод опирается на качественную теорию бесконечномерных динамических систем.
\end{abstract}

Ключевые слова: нелинейная краевая задача, теория аэроупругости, устойчивость, периодическое решение, панельный флаттер.

\section{NONLINEAR PANEL FLUTTER. BOLOTIN'S PROBLEM IN THE PRESENCE OF VISCOUS FRICTION}

\author{
(C) 2020 A. S. ZAPOV
}

\begin{abstract}
In this paper, we consider a nonlinear boundary-value problem proposed as the simplest model for describing oscillations in a gas flow. We analyze the stability of the zero equilibrium state and find a critical value of the velocity of the incoming gas flow. Exact solutions of the problem are found in the form of time-periodic functions and their stability is examined. All the results are obtained analytically based on the qualitative theory of infinite-dimensional dynamical systems.
\end{abstract}

Keywords and phrases: nonlinear boundary-value problem, theory of aeroelasticity, stability, periodic solution, panel flutter.

AMS Subject Classification: 34K18, 35G30, 37G35

1. Введение. В работе рассматривается краевая задача, которая была предложена в известной монографии В. В. Болотина [1] в качестве модели при описании колебаний пластин в сверхзвуковом потоке газа (воздуха). Данная краевая задача-упрощенный вариант постановки задач теории упругой устойчивости, посвященных колебаниям тел в потоке газа или жидкости. Такой вариант задачи, согласно В. В. Болотину, может возникнуть, если длина упругого тела достаточно велика и ее можно считать равной бесконечности. Анализ полученной в результате такой постановки задачи содержится в работе [3]. В представленной работе предложено существенное дополнение - в краевой задаче учтено вязкоупругое трение и добавлены соответствующие члены в уравнение, описывающее колебания такой пластины (см. работы $[2,4-8,10])$.

Ниже, в следующем разделе, рассмотрена уже математическая часть постановки задачи, т. е. будут приведены уравнения в нормированном и, конечно же, обезразмеренном виде. 
Подчеркнем, что в работе не предполагается использование метода Галеркина для анализа соответствующей краевой задачи. В работе рассмотрен вариант краевых условий, который предполагается в качестве основного в монографии В. В. Болотина. В работе будет изучен вопрос об устойчивости состояний равновесия $u \equiv 0$, а также найдем автомодельные $t$-периодические решения.

2. Постановка задачи. В работе будет рассмотрено дифференциальное уравнение с частными производными

$$
w_{t t}+g_{1} w_{t}+g_{2} w_{t, x x x x}+w_{x x x x}-b w_{x x}+d w+c w_{x}=F\left(w_{x x}, w_{x}, w_{x t}\right)
$$

вместе с краевыми условиями

$$
\begin{gathered}
w(t, x+2 \pi)=w(t, x), w_{x}(t, x+2 \pi)=w_{x}(t, x), \\
w_{x x}(t, x+2 \pi)=w_{x x}(t, x), w_{x x x}(t, x+2 \pi)=w_{x x x}(t, x),
\end{gathered}
$$

где $w=w(t, x)$ - функция, зависящая от пространственной переменной $x$ и времени $t$. Следуя работам $[2,7,8,10]$ будем считать, что нелинейность $F$ выбрана следующим образом:

$$
F\left(w_{x x}, w_{x}, w_{x t}\right)=\frac{q_{1}}{2 \pi} w_{x x} \int_{0}^{2 \pi}\left(w_{x}\right)^{2} d x+\frac{q_{2}}{2 \pi} w_{x x} \int_{0}^{2 \pi} w_{x t} w_{x} d x .
$$

Наконец, $g_{1}, g_{2}, d, c$ - некоторые положительные постоянные, а $b \in \mathbb{R}$. Основной параметрэто приведенная скорость набегающего потока газа (воздуха) $c$. В монографии [1] данная краевая задача (1), (2) предложена в качестве простейшей математической модели флаттера удлиненной пластинки, обтекаемой сверхзвуковым потоком газа. Отметим, что в монографии [1], а также в работе [3] она была изучена при дополнительном предположении, что $g_{2}=0$. Последнее предположение означает, что в задаче не учитывается вязкое трение. В настоящее время принято считать необходимым учет этого фактора при анализе задач теории упругой устойчивости (см. работы $[2,4-8,10])$.

Если краевую задачу (1), (2) дополнить до начально-краевой, т. е. краевую задачу (1), (2) дополнить начальными условиями

$$
w(0, x)=f(x), \quad w_{t}(0, x)=g(x),
$$

где функции $f(x), g(x)$ по переменной $x$ имеют период $2 \pi$, а также при $x \in[0,2 \pi]$ принадлежат пространствам Соболева $W_{2}^{4}[0,2 \pi], W_{2}^{2}[0,2 \pi]$ соответственно. Напомним, что функция $h(x) \in$ $H_{2}^{s}[0,2 \pi]$, если $h(x) \in L_{2}(0,2 \pi)$ и ее производные $h^{\prime}(x), h^{\prime \prime}(x), h^{\prime \prime \prime}(x), h^{\prime \prime \prime \prime}(x) \in L_{2}(0,2 \pi)$. В силу теории вложения $h(x) \in C^{s-1}[0,2 \pi]$.

При таком выборе $f(x), g(x)$ начально-краевая (смешанная) задача $(1),(2),(3)$ будет локальноразрешима. (см. $[7,9])$. В частности, смешанная задача $(1),(2),(3)$ имеет решение при $t \in\left[0, t_{0}\right]$, $t_{0}>0$. Такое решение зависит от начальных условий и параметров задачи.

Все вышесказанное позволяет при анализе динамики решений использовать также методы теории динамических систем, качественной теории дифференциальных уравнений, а также изучить устойчивость состояний равновесия, периодических решений.

3. Анализ линеаризованной краевой задачи. Для изучения вопроса об устойчивости нулевого состояния равновесия следует рассмотреть линеаризованный вариант краевой задачи (1), (2). Выпишем соответствующую краевую задачу:

$$
\begin{gathered}
w_{t t}+g_{1} w_{t}+g_{2} w_{t, x x x x}+w_{x x x x}-b w_{x x}+d w+c w_{x}=0, \\
w(t, x+2 \pi)=w(t, x), w_{x}(t, x+2 \pi)=w_{x}(t, x), \\
w_{x x}(t, x+2 \pi)=w_{x x}(t, x), w_{x x x}(t, x+2 \pi)=w_{x x x}(t, x) .
\end{gathered}
$$

Линейная краевая задача (4), (5) допускает нетривиальный набор решений вида $w(t, x)=$ $\exp \left(\lambda_{n} t+i n x\right)$, где $\lambda_{n}$, в свою очередь, удовлетворяет характеристическому уравнению

$$
\lambda^{2}+G_{n} \lambda+K_{n}+i n c=0,
$$


где $G_{n}=g_{1}+g_{2} n^{4}, K_{n}=n^{4}+b n^{2}+d, n=0, \pm 1, \pm 2 \ldots$

Совокупность корней семейства алгебраических уравнений (6) формирует спектр устойчивости решений краевой задачи (4), (5). Пусть далее $b \geqslant 0$. Если допустить, что величина $b$ может быть отрицательной, то не исключен вариант, при котором $\lambda_{p}=0$ при некотором натуральном значении $p$, если даже $c=0$. Последний вариант приводит уже нас к иной математической задаче. Если при этом $c=0$, то можно утверждать, что из выполнения условий $\operatorname{Re} \lambda_{n}<0, n= \pm 1, \pm 2 \ldots$ вытекает асимптотическая устойчивость решений линейной краевой задачи $(4),(5)$ и нулевого решения уже нелинейной краевой задачи (1), (2). При существовании такого $n=n_{0}$, что $\operatorname{Re} \lambda_{n}>0$ можно сделать вывод о неустойчивости решений линейной системы (4), (5) и неустойчивости нулевого решения краевой задачи (1), (2). При $n=0$ оба корня характеристического уравнения (6) имеют отрицательную действительную часть. Подчеркнем при этом, что семейство функций $\exp ($ in $x)$ является базисом в пространстве $L_{2}(0,2 \pi)$.

Пусть выполнены неравенства $\operatorname{Re} \lambda_{n} \leqslant 0, n= \pm 1, \pm 2 \ldots$ и вместе с этим существует по крайней мере одно $n=k$, такое, что $\operatorname{Re} \lambda_{k}=0$. В таком случае в задаче об устойчивости нулевого решения нелинейной краевой задачи (1), (2) реализуется критический случай.

Для анализа расположения корней характеристического уравнения (6) используем стандартный алгебраический прием, позволяющий свести уравнение с комплексными коэффициентами к уравнению четвертой степени с действительными коэффициентами с последующим использованием критерия Рауса-Гурвица. Для этого, как хорошо известно, следует рассмотреть вспомогательное алгебраическое уравнение с комплексными коэффициентами $\lambda^{2}+G_{n} \lambda+\left(K_{n}-i n c\right)=0$, а также новое характеристическое уравнение

$$
\left(\lambda^{2}+G_{n} \lambda+K_{n}+i n c\right)\left(\lambda^{2}+G_{n} \lambda+K_{n}-i n c\right)=0,
$$

где $G_{n}=g_{1}+g_{2} n^{4}, K_{n}=n^{4}+b n^{2}+d, n= \pm 1, \pm 2 \ldots$ Очевидно, что неравенства $\operatorname{Re} \lambda_{n}<0$ выполнены (или не выполнены) для уравнений (6) и (7) одновременно. В свою очередь, для анализа расположения корней уравнения (7) можно применить критерий Рауса-Гурвица. Для этого перепишем левую часть уравнения в виде следующего многочлена:

$$
\begin{gathered}
R_{n}(\lambda)=\lambda^{4}+a_{1} \lambda^{3}+a_{2} \lambda^{2}+a_{3} \lambda+a_{4}, \\
a_{1}=a_{1}(n)=2 G_{n}, \quad a_{2}=a_{2}(n)=G_{n}^{2}+2 K_{n}, \\
a_{3}=a_{3}(n)=2 G_{n} K_{n}, \quad a_{4}=a_{4}(n)=K_{n}^{2}+n^{2} c^{2} .
\end{gathered}
$$

Подчеркнем, что при $n=0$ справедливо неравенство $\operatorname{Re} \lambda_{0(1,2)}<0$ и, кроме этого, коэффициенты характеристического уравнения (7) (после упрощений) зависят только от $n^{2}$ (четные функции $n)$. Поэтому дальнейший анализ расположения корней уравнения (7) можно и целесообразно проводить для $n=1,2,3 \ldots$ Анализ расположения корней многочлена $R_{n}(\lambda)$ с использованием критерия Рауса-Гурвица приводит к необходимости проверки неравенств

$$
a_{1}>0, \quad a_{2}>0, \quad a_{3}>0, \quad a_{4}>0,
$$

которые, разумеется, выполняются при всех $n$, а также

$$
\Delta_{2}=a_{1} a_{2}-a_{3}>0, \quad a_{3} \Delta_{2}-a_{4} a_{1}^{2}>0,
$$

из которых содержательно лишь одно и может быть записано в виде

$$
\frac{K_{n} G_{n}^{2}}{n^{2}}-c^{2}>0, \quad n= \pm 1, \pm 2 \ldots
$$

В частности, если

$$
c^{2}=\min c_{n}^{2}, \quad c_{n}^{2}=\frac{K_{n} G_{n}^{2}}{n^{2}},
$$

то при таких $c$ нулевое решение асимптотически устойчиво.

Теорема 1. Существует такое $c=c_{*}>0$, что при $c<c_{*}$ нулевое состояние краевой задачи (1), (2) асимптотически устойчиво, а при $c>c_{*}$ теряет свою устойчивость. В случае 
$c=c_{*}$ реализуется критический случай в задаче об устойчивости. При этом

$$
c_{*}=\min c_{n}, \quad c_{n}=\frac{g_{1}+g_{2} n^{4}}{n} \sqrt{n^{4}+b n^{2}+d}, \quad n= \pm 1, \pm 2 \ldots
$$

Естественно, что достаточно ограничиться случаем натуральных значений $n$, так как $c_{-n}=c_{n}$. Доказательство данного утверждения основано на том, что последовательность

$$
c_{n}=\left(g_{1}+g_{2} n^{4}\right) \sqrt{n^{2}+b+\frac{d}{n^{2}}}>0
$$

при всех натуральных значениях $n$ и имеет наименьшее значение.

Отметим, что при $g_{2}=0$ последовательность $c_{n}$ достигает своего минимума на номере $n=n_{0}$ (и, конечно же, $n=-n_{0}$ ), где $n_{0}$, в свою очередь, равно одному из двух значений: $j_{0}$, либо $j_{0}+1$, где $j_{0}=\operatorname{entier}(\sqrt[4]{d})$. Если при этом значение $d$ достаточно мало, то очевидно, что $n_{0}=1$. Аналогичный факт имеет место при малых $d$, если $g_{2}>0$. В остальных случаях требуется более детальный анализ, но не исключен вариант, при котором $n_{0}$ может принимать значения, отличные от 1 .

Условие устойчивости $c<c_{*}$ может быть записано в иной форме, которая менее наглядная с точки зрения приложений, но относительно содержательна для последнего раздела данной работы. Элементарные преобразования показывают, что условие $c<c_{*}$ эквивалентно системе неравенств

$$
H_{n}<0, \quad H_{n}=\frac{c^{2}}{G_{n}^{2}}-\frac{K_{n}}{n^{2}}
$$

при всех $n \in \mathbb{N}$. Подчеркнем, что при больших значениях $n$ неравенство с номером $n$ выполнено $\left(\lim _{n \rightarrow \infty} H_{n}=-\infty\right)$.

4. Об автомодельных периодических решениях нелинейной краевой задачи. Возвратимся теперь к анализу задачи в нелинейной постановке, т. е. анализу краевой задачи (1), (2). Предположим, что $w=w(t, x)$ - решение данной краевой задачи. Тогда такое решение можно представить в виде ряда

$$
w(t, x)=\sum_{n=-\infty}^{\infty} w_{n}(t) \exp (\text { in } x)
$$

В свою очередь, коэффициенты ряда (8) удовлетворяют следующей бесконечной системе обыкновенных дифференциальных уравнений

$$
w_{n}^{\prime \prime}+G_{n} w_{n}^{\prime}+\left(K_{n}+i c n\right) w_{n}=-n^{2}\left(q_{1} \sum_{m=-\infty}^{\infty} m^{2}\left|w_{m}\right|^{2}+q_{2} \frac{d}{d t} \sum_{m=-\infty}^{\infty} m^{2}\left|w_{m}\right|^{2}\right) w_{n}
$$

где $w_{n}=\bar{w}_{n}, n=0, \pm 1, \pm 2 \ldots$ Покажем, что система (9) может иметь решение следующего вида:

$$
w_{k}=\eta_{k} \exp \left(i \omega_{k} t\right), \quad w_{-k}=\eta_{k} \exp \left(-i \omega_{k} t\right), \quad \omega_{k} \in \mathbb{R}
$$

a $w_{n}=0$, если $n \neq k$. Отметим также, что можно считать $w_{0}=0$. Действительно, при $n=0$ уравнение (9) приобретает вид

$$
w_{0}^{\prime \prime}+G_{0} w_{0}^{\prime}+K_{0} w_{0}=0,
$$

где $G_{0}=g_{1}, K_{0}=d$. В получившемся уравнении все его решения будут стремиться к нулю.

Без нарушения общности можно считать, что $\eta_{k}>0$. После подстановки функций (10) в уравнение (8) получим уравнение для определения $\omega_{k}$ и $\eta_{k}$ :

$$
-\omega_{k}^{2}+\left(g_{1}+g_{2} k^{4}\right) i \omega_{k}+\left(k^{4}+b k^{2}+d+i c k\right)=-2 k^{4} q_{1}\left|\eta_{k}\right|^{2}, \quad\left|\eta_{-k}\right|=\left|\eta_{k}\right| .
$$

Последнее уравнение эквивалентно следующей системе алгебраических уравнений:

$$
\begin{gathered}
-\omega_{k}^{2}+\left(k^{4}+b k^{2}+d\right)=-2 q_{1} k^{4} \eta_{k}^{2}, \\
\left(g_{1}+g_{2} k^{4}\right) \omega_{k}+c k=0,
\end{gathered}
$$


откуда следует, что

$$
\omega_{k}=-\frac{k c}{g_{1}+g_{2} k^{4}}, \quad \eta_{k}^{2}=\frac{1}{2 k^{2} q_{1}} \cdot \frac{1}{\left(g_{1}+g_{2} k^{4}\right)^{2}}\left(c^{2}-c_{k}^{2}\right), \text { где } c_{k}^{2}=\frac{\left(g_{1}+g_{2} k^{4}\right)^{2}}{k^{2}}\left(k^{4}+b k^{2}+d\right) .
$$

Таким образом, решение с номером $k$ существует, если выполняется неравенство $c^{2}-c_{k}^{2}>0$ или же $c>c_{k}$.

Теорема 2. Пусть $c>c_{k}$. Тогда краевая задача (1), (2) имеет автомодельное периодическое решение

$$
w_{k}(t, x)=\eta_{k}\left(\exp \left(i \omega_{k} t+i k x+i \phi_{k}\right)+\exp \left(-i \omega_{k} t-i k x-i \phi_{k}\right)\right)
$$

Напомним, что $c_{k}>c_{*}$, т. е. периодические решения появляются при превышении $c$ критической скорости флаттера $c_{*}$ и, следовательно, потере устойчивости нулевого решения краевой задачи (1), (2).

Следствие 1. Пусть $c<c_{k}$. Тогда, как уже отмечалось, существует решение с номером $k$. Если существует набор $c_{j 1}, \ldots c_{j p}$ таких, что

$$
c_{j s} \leqslant c_{k}, \quad s=1, \ldots, p
$$

то в таком случае существуют и автомодельные решения с соответствующими номерами $j_{1}, j_{2}, \ldots, j_{p}$

Замечание 1. Формулу для амплитуды можно переписать в иной форме:

$$
\eta_{m}^{2}=\frac{1}{2 q_{1} m^{2}} H_{m}, \quad H_{m}=\frac{c^{2}}{G_{m}^{2}}-\frac{K_{m}}{m^{2}},
$$

т. е. необходимо выполнение неравенства $H_{m}>0$.

5. Анализ устойчивости автомодельных периодических решений. Одномодовому решению $w_{m}(t, x)$ из предыдущего раздела соответствует решение системы дифференциальных уравнений (9), для которого компонента с номером $m$ будет иметь вид

$$
w_{m}(t)=v_{m}(t)=\eta_{m} \exp \left(i \omega_{m} t\right)
$$

где

$$
\omega_{m}=-\frac{m c}{g_{1}+g_{2} m^{4}}, \eta_{m}^{2}=\frac{1}{2 q_{1} m^{2}} \cdot \frac{1}{\left(g_{1}+g_{2} m^{4}\right)^{2}}\left(c^{2}-c_{m}^{2}\right),
$$

а остальные компоненты $w_{n}(t)=0$ при $n \neq m$. Поэтому положим

$$
w_{n}(t)=u_{n}(t), \quad w_{m}(t)=v_{m}(t)\left(1+u_{m}(t)\right) .
$$

В результате замены (12) получим новую систему уравнений, у которой следует исследовать устойчивости нулевого решения. Для этого рассмотрим ее линеаризованный вариант. После стандартных преобразований линеаризованная система будет выглядеть следующим образом:

$$
\begin{gathered}
u_{m}^{\prime \prime}+u_{m}^{\prime}\left(2 i \omega_{m}+G_{m}\right)+2 q_{1} m^{4}\left(u_{m}+\bar{u}_{m}\right)+2 q_{2} m^{4}\left(u_{m}^{\prime}+{\overline{u_{m}}}^{\prime}\right)=0, \quad n=m \\
u_{n}^{\prime \prime}+u_{n}^{\prime}\left(2 i \omega_{n}+G_{n}\right)+u_{n} Q_{n, m}=0, \quad n \neq m
\end{gathered}
$$

где $Q_{n, m}=K_{m}+i n c-\omega_{n}^{2}+i G_{n} \omega_{n}+2 q_{1} n^{2} m^{2} \eta_{m}^{2}$.

Напомним, что $\omega_{n}=-\frac{c n}{G_{n}}$, т. е. после упрощений получим, что

$$
Q_{n, m}=n^{2}\left(H_{m}-H_{n}\right), \quad H_{n}=\frac{c^{2}}{G_{n}^{2}}-\frac{K_{n}}{n^{2}}
$$

Автомодельное решение $v_{m}(t)=\eta_{m} \exp \left(i \omega_{m} t\right)$ устойчиво, если для каждого из дифференциальных уравнений (14) нулевое решение асимптотически устойчиво. Наконец, нулевое решение уравнения (13) устойчиво. 
Начнем с анализа устойчивости нулевого решения у последовательности уравнений (14). Рассмотрим уравнение с номером $n(n \neq m)$. Его нулевое решение асимптотически устойчиво, если корни квадратного уравнения

$$
\lambda^{2}+\left(G_{n}+2 i \omega_{n}\right) \lambda+Q_{n, m}=0
$$

лежат в левой полуплоскости комплексной плоскости. Из критерия Рауса-Гурвица следует, что необходимым и достаточным условием для этого служит система двух неравенств

$$
G_{n}>0, \quad Q_{n, m}>0 .
$$

Напомним, что $G_{n}=g_{1}+g_{2} n^{4}>0$ при всех значениях $n$. Поэтому остается одно неравенство $Q_{n, m}>0$, которое можно записать в иной форме:

$$
H_{n}<H_{m}, \quad H_{n}=\frac{c^{2}}{G_{n}^{2}}-\frac{K_{n}}{n^{2}} .
$$

Неравенство (15) при $n>n_{0}$ очевидно, т. к. $\lim _{n \rightarrow \infty} H_{n}=-\infty$. Последнее неравенство должно быть выполнено при всех $n \neq m, m \in \mathbb{N}$.

Перейдем к анализу устойчивости нулевого решения уравнения (13). Для анализа устойчивости положим $u_{m}=y_{m}+i z_{m}$, т. е. комплекснозначную функцию $u_{m}(t)$ представим в развернутом виде.

В результате получим систему дифференциальных уравнений для $y_{m}(t), z_{m}(t)$ следующего вида:

$$
\begin{gathered}
y_{m}^{\prime \prime}+y_{m}^{\prime} G_{m}-2 \omega_{m} z_{m}^{\prime}+4 q_{2} m^{4} \eta_{m}^{2} y_{m}^{\prime}+4 q_{1} m^{4} \eta_{m}^{2} y_{m}=0 \\
z_{m}^{\prime \prime}+z_{m}^{\prime} G_{m}+2 \omega_{m} y_{m}^{\prime}=0
\end{gathered}
$$

В системе дифференциальных уравнений (16) соответствующей номеру $m$ можно упростить запись некоторых коэффициентов. Так, например,

$$
4 q_{2} m^{4} \eta_{m}^{2}=2 \frac{q_{1}}{q_{2}} m^{2} H_{m}, \quad 4 q_{1} m^{2} \eta_{m}^{2}=2 m^{2} H_{m}, \quad H_{m}=\frac{c^{2}}{G_{m}^{2}}-\frac{K_{m}}{m^{2}},
$$

как и ранее. Характеристический многочлен соответствующей системы дифференциальных уравнений (16), как следует из стандартных вычислений, имеет следующий вид:

$$
\left(\lambda^{2}+\left(G_{m}+2 q m^{2} H_{m}\right) \lambda+2 m^{2} H_{m}\right)\left(\lambda^{2}+G_{m} \lambda\right)+4 \omega_{m}^{2} \lambda^{2}=0, \quad q=\frac{q_{1}}{q_{2}}>0 .
$$

Один из корней характеристического уравнения $\lambda_{1}=0$, а все остальные его корни лежат в левой полуплоскости, что проверяется при помощи критерия Рауса-Гурвица, примененного к уравнению

$$
\lambda^{3}+S_{m} \lambda^{2}+J_{m} \lambda+L_{m}=0
$$

где

$$
\begin{gathered}
S_{m}=2\left(G_{m}+2 q H_{m}\right)>0, \quad L_{m}=2 m^{2} H_{m} G_{m}>0 \\
J_{m}=4 \omega_{m}^{2}+G_{m}\left(G_{m}+2 q m^{2} H_{m}\right)+2 m^{2} H_{m}^{2}>0,
\end{gathered}
$$

при любом выборе параметров задачи, когда существует автомодельное решение с номером $m$ $\left(H_{m}>0\right.$ - один из вариантов условий существования решения с номером $\left.m\right)$. При этом справедливость неравенства $S_{m} J_{m}>L_{m}$ очевидна. Из последних четырех неравенств вытекает, что все корни уравнения (17) лежат в левой полуплоскости комплексной плоскости. Последний вывод является следствием из критерия Рауса-Гурвица. В свою очередь, из теоремы Андронова-Витта вытекает, что периодическое решение $v_{m}(t)$, как решение системы (13) устойчиво (орбитально асимптотически устойчиво).

Итак, справедливо следующее утверждение.

Теорема 3. Автомодельное периодическое решение $v_{m}(t)=\eta_{m} \exp \left(i \omega_{m} t\right)$, где

$$
\omega_{m}=-\frac{c m}{G_{m}}, \quad \eta_{m}^{2}=\frac{1}{2 q_{1} m^{2}} H_{m}
$$


устойчиво, если при всех натуральных значениях $n \neq m$ выполнены неравенства

$$
H_{m}>H_{n} .
$$

Подчеркнем, что $H_{m}>0$ (условие существования цикла), а

$$
H_{n}=\frac{c^{2}}{G_{n}^{2}}-\frac{K_{n}}{n^{2}} .
$$

Следствие 2. Из условий устойчивости (18) вытекает, что

$$
H_{m}=\max H_{n} .
$$

Следовательно, условие (19) - одна из форм записи условий устойчивости иикла с номером т.

6. Заключение. В работе приведены условия устойчивости нулевого состояния равновесия и указана критическая скорость $c_{*}$, превышение которой приводит к потере устойчивости, а также найдены периодические решения рассматриваемой краевой задачи. Эти одномодовые решения описывают колебания пластины следующим образом:

$$
w_{k}(t, x)=\eta_{k}\left(\exp \left(i \omega_{k} t+i k x+i \phi_{k}\right)+\exp \left(-i \omega_{k} t-i k x-i \phi_{k}\right)\right)=2 \eta_{k} \cos \left(\omega_{k} t+k x+\phi_{k}\right), \quad \phi_{k} \in \mathbb{R},
$$

то есть такие решения имеют форму бегущей волны.

В заключение подчеркнем, что условия существования и устойчивости были записаны в двух формах. Напомним одну из них. Решение с номером $m$ существует, если $H_{m}>0$, и оно устойчиво, если

$$
H_{m}=\max H_{n}, \quad n=1,2,3 \ldots
$$

и, естественно, этот максимум положителен.

\section{СПИСОК ЛИТЕРАТУРЫ}

1. Болотин В. В. Неконсервативные задачи теории упругой устойчивости. - М.: Наука, 1961.

2. Запов A. C. Об одной математической модели в теории упругой устойчивости// Вестн. Удмурт. ун-та. Мат. Мех. Комп. науки. - 2019. - 1, № 29. - С. 29-39.

3. Куликов A. Н. Аттракторы одной нелинейной краевой задачи, встречающейся в теории аэроупругости// Диффер. уравн. - 2001. - 3, № 37. - С. 397-401.

4. Куликов А. Н. Бифуркация автоколебаний пластинки при малом демпфировании в сверхзвуковом потоке газа// Прикл. мат. мех. - 2009. - 2, № 73. - С. 271-281.

5. Куликов А. Н. О возможности реализации сценария Ландау-Хопфа перехода к турбулентности в двух задачах теории упругой устойчивости// Диффер. уравн. - 2011. - 1, № 47. - С. 296-298.

6. Куликов А. Н. О реализации сценария Ландау-Хопфа перехода к турбулентности в некоторых задачах теории упругой устойчивости// Диффер. уравн. - 2012. - 9, № 48. - С. 1278-1291.

7. Куликов A. Н. Некоторые бифуркационные задачи теории упругой устойчивости и математической физики/ Диссертация на соискание уч. степ. докт. физ.-мат. наук - Н. Новгород, 2018.

8. Томпсон Дж. М. Т. Неустойчивости и катастрофы в науке и технике. - М.: Мир, 1985.

9. Якубов С. Я. Разрешимость задачи Коши для абстрактных квазилинейных гиперболических уравнений второго порядка и их приложения// Тр. Моск. мат. о-ва. - 1970. - 23. - C. 37-60.

10. Paidoussis M. P., Issid N. T. Dynamic stability of pipes conveying fluid// J. Sound Vibr. - 1974. - 3, № 33. - P. 267-294.

Запов Александр Сергеевич

Ярославский государственный университет им. П. Г. Демидова

E-mail: yar.promo.zapov.a@gmail.com 